\title{
Site Evaluation: The Second Crucial Phase of Startup in Clinical Research
}

\author{
Article by Vinod Gyanchandani ${ }^{1}$, Pradnya Mahatme ${ }^{2}$, \& Ashok Motwani ${ }^{3}$ \\ ${ }^{1}$ Global Drug Development Experts, Nagpur, Maharashtra, India \\ E-mail:vgyanchandani@gddexperts.com \\ ${ }^{2}$ Global Drug Development Experts, Nagpur, Maharashtra, India. \\ E-mail:pmahatme@gddexperts.com \\ ${ }^{3}$ Sindhu Mahavidyalaya, Nagpur, Maharashtra, India \\ E-mail: ashokkumarmotwani@gmail.com
}

\begin{abstract}
This review article presents an overview of the 'site evaluation' process, the roles and responsibilities of participants, the standard requirements and processes for activities which needs attention, and the importance of communication and flexibility during this startup phase of the study. This article is written in accordance with International Conference on Harmonisation of Technical Requirements for Registration of Pharmaceuticals for Human Use Harmonized Tripartite Guideline for Good Clinical Practice (ICH GCP Topic E6).
\end{abstract}

Keywords: Protocol, Sponsor, Investigator, Site, Institutional Ethics Committee, Study team

Clinical Research refers to a systematic investigation in human subjects for evaluating the safety and efficacy of any new drug. In today's scientific era, research is taking a major stride in all streams and newer and better drugs are being introduced to cure ailments, which are difficult to treat. Clinical trials are the mainstay for bringing out new drugs to the market and constitute approximately $70 \%$ of the total time and money spent in drug discovery process under which, Sponsor mainly provides the funding, investigational product and the protocol with the objective of getting a Regulatory approved drug or device onto the market; and the Investigator at the site is the qualified and trained individual who accepts full responsibility to conduct the trial according to the protocol, to protect the subjects right and safety and to collect and submit credible data to the Sponsor.

\section{Introduction}

Implementation and conduct of a clinical study can be a complex process that involves a team from various disciplines and multiple steps that are dependent on one another. This article offers guidance for navigating the study start-up and activation process.

The startup phase can be one of the most critical intervals in the conduct of the trial, as it requires targeted strategies from both the Sponsor and the site. Regardless of which strategies are chosen to accomplish these tasks, clear and transparent communication and flexibility to accommodate changes are some of the keys to a successful study startup. The processes involved with study startup are complex, and initially include site identification, qualification, and evaluation followed by negotiating contracts, budgets, regulatory approvals, and trial registrations on regulatory portal.

The Site evaluation visit is a meeting conducted at a potential trial site, to introduce a prospective Investigator to the trial protocol, to familiarize the Investigator with regulatory obligations, and to assess the Investigator's qualification, experience, expertise and interest in the trial and ability to carry out the trial in accordance with ICH-GCP and regulatory requirements. During this visit, the CRA also assess that, whether the infrastructures and facilities available at the site are adequate/inadequate to conduct the study.

The site evaluation is a critical step for both the Sponsor and the site. For the Sponsor, it is the primary method of determining the experience, expertise and interest of the Investigator, as well 
as the overall capability of the site to conduct their study. For the site, evaluation visit determines whether or not, the site will be selected to participate in the research, hence it is very important for the Investigator and the site to be well prepared for a site evaluation visit and ready to show their site, at its best.

Both Sponsors and sites need to be flexible while completing study startup activities

Before the evaluation visit, the Sponsor/ CRO representative (here-in after refer to as CRA) will:

1. Request the Investigator for potential meeting dates and time to accommodate as many key personnel as possible.

2. Discuss with the Investigator that all required team members will be available and have allocated sufficient time for the meeting date.

3. Ensure that the key site personnel receive copies of the protocol, Investigator's brochure, and any other study materials for review and comment.

4. Ensure that the appointment with the Investigator is confirmed.

5. Carefully review the site evaluation checklist for assessing the site, the advantage of using a checklist is that the CRA won't inadvertently forget to assess some important items.

Site evaluation checklist will be mainly based on following factors:

- Investigator's qualification, experience, expertise and interest in the trial

- Availability of the basic infrastructure and other required facilities for the study

- Availability of sub-Investigator and other required team members for the study

- Enrolment potential of the site which in turn is linked to number of patients seen by the Investigator for the disease condition under study

- Investigator's commitment and willingness to spare time for the trial

- Availability of an Institutional Ethics Committee having the composition and written operating procedures in compliance with the applicable regulatory requirements

- Source documentation practices of the site

- Administrative policies related to the execution of clinical trial agreement

During the evaluation visit, the CRA will have a face-to-face meeting with the principal Investigator (PI) and the study team to discuss the test product, study protocol, study execution methods, and all other required information to be discussed and exchanged during this visit; to ensure understanding of the product use and also the site clearly and fully understand and accept their obligations in conducting clinical trials. CRA also assesses the feasibility of conducting the study at this site, by considering, the time and resources, infrastructure, potential subject availability and ease of implementing the study at the site. The interest of the Investigator will also be determined and the CRA may have the responsibility for determining which Investigators and sites will participate in the clinical study.

The ultimate goal of Evaluation visit is:

1) Ensure the selection of right Investigator and

2) Ensure the capability of site to conduct the trial.

\section{Selection of investigator}

While performing the selection of Investigator; following below aspects are discussed and performed with the Potential Investigator:

\subsection{Site staff review}

Conduct of a clinical trial requires team effort and involvement of personnel with delegation of specific duties. During the site evaluation visit, the CRA will evaluate whether the investigator/ site has sufficient study team to do the study. Not only there must be appropriate staff available for a study, but they must have sufficient time to do the necessary work required for the study. Site staff varies as per study design but the various personnel that constitutes the 
study team at a trial site mainly include, Principal Investigator; Sub- Investigator, Clinical Research Coordinator, Study Nurses, phlebotomist, Pharmacist etc.

A Principal investigator (PI) is the physician who leads the conduct of a clinical trial. The leadership role of the PI is the foundation that creates the successful clinical trial. The PI is responsible for all clinical research activities at the center and have the ultimate responsibility for the subject's safety in a clinical trial. The regulatory authorities mandate the PI to sign a FDA form 1572/ Investigator Undertaking that outlines the specific responsibilities a doctor must accept as leader of the clinical investigation.

In the Investigator selection process, it is important to remember that, the Sponsor is entrusting this individual to research their investigational product that will result in quality, reproducible trial data. Most importantly, this individual will be managing the study subjects' safety and medical care during the trial and hence, must have proper credentials and specialty experience to do so.

To confirm the same, the Sponsor will review the current curriculum vitae (CV) and/or other relevant documentation of potential Investigator in order to make a general assessment of the Investigator's experience and expertise. Conversing with the Investigator in person will allow the Sponsor to determine his or her research activity, especially in the therapeutic area of interest. The CRA will also want to know if the Investigator has conducted trials similar to the one being proposed, or has worked with similar compounds.

\section{General recommendation to assess the experience and expertise of investigator}

- The Investigator(s) should be qualified by education as documented by board certifications, have adequate training via academic or clinical practice, recent publications or presentations, professional memberships, etc., in related areas; aware of, and comply with, GCP and finally have experience in clinical research and human clinical trials of the disease under study, to assume responsibility, for the proper conduct of the clinical trial.

- Not listed on FDA's Disqualified/Restricted Investigators lists, Administrative Actions list, or similar local/national/international regulatory authority debarred lists.

- Thoroughly familiar with the appropriate use of the investigational product(s), as described in the clinical protocol, in the current Investigator's Brochure, in the product information and in other information sources provided by the Sponsor.

- Privilege to conduct clinical trial at an institution; meeting the selection criteria as investigational site.

- Sufficient patients to enroll the target number of study patients in the necessary time frame.

- Adequate time for the administration of the study, adequate support including a clinical research coordinator and other qualified persons to whom the Investigator could delegate significant trial-related duties and adequate resources, including space, for data reporting and storage

- Willingness to obtain and maintain Institutional Ethics Committee and scientific committee review and approval for the study, as necessary, and to abide by their other requirements.

- Willingness to sign an Investigator's agreement specifying responsibilities and a confidentiality agreement specifying that the Investigator will maintain propriety information in confidence regarding the product being investigated, the clinical study design, and the clinical study results, unless the Sponsor gives permission to disclose such information.

- Willingness to provide and sign information required on financial disclosure forms.

- Willingness to allow monitoring and auditing by the Sponsor representatives, inspection by the Institutional Ethics Committee, and the appropriate regulatory authorities which includes access to the hospital records, Investigator study files, and patient files as they pertain to the study. 
South American Journal of Clinical Research

Volume 3, Issue 1, 2016

- Hence Investigators are selected from their work on previous clinical trials, publications and referrals and they should meet the study-specific criteria as stated in the study protocol.

\section{General recommendation to assess the interest level of investigator}

Conversing with the Investigator in person will allow the CRA to determine the interest level of the Investigator. While listening to the answers to CRAs questions, CRA should also be aware of the non-verbal clues to the Investigator's interest in your project. Is the Investigator actively listening? Asking pertinent questions? Being attentive to the conversation and materials? If an Investigator professes great interest in doing a study, but is also getting and replying to his email during the meeting — obviously his interest level isn't very high. One of the most critical factors for the success of a study is the interest of the Investigator. If the Investigator isn't truly interested, the study won't be foremost in his or her mind and it will probably suffer because of it.

The CRA will also want to assess the Investigator's reactions to the protocol. Is there anything in the protocol that the physician objects to doing? Is the Investigator willing to follow the protocol as it is written? Is the physician comfortable with the study design, and the use of a placebo, if applicable? Does the protocol match his or her generally accepted clinical practice, except for study-specific parameters? Making assumptions that everything is acceptable without checking is a risky path to follow. It's better to find out about potential problems and conflicts before the study is offered to him or her.

This is also a good time to review the following differences between clinical practice and clinical research with the Investigator. This is particularly important if he or she is new or relatively inexperienced. Failure to understand these differences can lead to protocol violations, which could have serious impact on the quality of the study, or lead to its termination.

Some of these differences are:

- During a clinical trial, the definitions used for adverse events are regulatory definitions, and are not necessarily based on usual clinical observation.

- Concomitant medications that would normally be prescribed for the subject may not be allowed by the protocol, or, if allowed, the dose and regime may differ from standard practice.

- The protocol treatment period for the disease being studied may differ from normal medical practice. It could be longer or shorter, and is very apt to involve more frequent visits.

- If the study is placebo controlled, the Investigator must be comfortable using placebo in subjects with the condition being studied.

\section{Additional site staff}

To successfully run a clinical trial, it is essential that an Investigator appoint certain ancillary staffs that are typically required to support the Pl. It mainly includes Sub-Investigators and Clinical Research Coordinator, Nurses, Pharmacist, Phlebotomist, etc. Ancillary staffs, plays a key role in the execution of the clinical trial and act as a direct support to the principal Investigator. Therefore careful consideration should be given to review each person's qualifications, their curriculum vitae (CVs), medical licenses and clinical trial experience of study staff.

Sub-investigator: Sub-investigator is an important member of the study team designated and supervised by the investigator to perform critical trial-related procedures and/or to make important trial-related decisions. Sub-investigator is required mainly to share investigator's responsibilities as well as to act as a back-up person when the investigator is not available. It should be stressed that sub Investigators will have the same regulatory responsibilities as the PI; but the principal Investigators must assure that they will oversee the entire clinical investigation including the involvement of the sub Investigators. If deemed appropriate by the investigator more than one co-investigator/sub-investigator can be included in the study team. The CRA 
should assess the qualifications of any sub Investigators or other physicians who will be evaluating the subjects.

Clinical research coordinator: Clinical research coordinator is a clinically oriented person employed at investigator's site and involved in the daily operations of a study as required by a protocol, to coordinate overall conduct of the trial under the supervision of the PI. This person often coordinates patient enrollment and visits, manages the study documentation, perform minor assessments and laboratory work (depends upon level of clinical skills), record and organize the data for transfer into Case Report Forms (CRF) in compliance with protocol, GCP and applicable regulatory guidelines. A dedicated clinical research coordinator is must for the smooth execution of a clinical trial

\subsection{Brief introduction to the Study}

CRA has to first ensure that the site has received essential study documents, such as Protocol and Investigator Brochure and any other study material required to discuss the study with the Investigator.

The Protocol contains a study plan on which all clinical trials are based. The plan is designed to safeguard the health of the participants as well as answer specific research questions. The protocol describes what types of people may participate in the trial, the schedule of tests, procedures, medications, and dosages, and the length of the study. Hence the Protocol is reviewed and discussed in detail with the Investigators and the site staff emphasizing on background, study rationale, protocol's objective, Inclusion/ exclusion criteria, study design, study procedures, end points, and statistical considerations of a clinical trial and all the requirements for an adequate and well-controlled study; this helps CRA to assess, the availability of time and resources at site. Any questions that arise related to study should be clarified and any comments and or suggestions from the site personnel about the protocol should be documented.

Investigators and site staff should thoroughly review the protocol, communicate any concerns to the CRA, and ensure they understand all of the CRA's responses. Lack of clarity at this point can lead to bigger issues later, including slow recruitment, lack of enrollment, and difficult follow up.

The Investigator brochure (IB) is also reviewed during this visit. The IB is a compilation of all of the nonclinical, preclinical, and clinical data collected to date on the investigational product. The Investigator must review and understand this document in detail, because it provides information on pharmacology, toxicology, and other pertinent data confirming that the starting dosage to be administered to the subjects is reasonably safe.

\subsection{Subject enrollment}

One of the primary problems, facing the smooth execution of clinical trial is, enrolling appropriate subjects within the allotted enrollment time. The CRA will assess, whether the Investigator has the potential for recruiting the required number of eligible subjects for the study in the proposed recruitment period. The CRA will thoroughly assess the enrollment potential of the site, including whether subjects will come from the Investigator's current patient population or if they will be drawn from elsewhere. Note that it is usually easier to assess enrollment potential for chronic disease studies than it is for acute disease studies. For chronic diseases, such as arthritis or diabetes, the Investigator should already have appropriate patients among his or her current patient base. It does not necessarily follow that these patients will qualify for or want to participate in the research study, but it gives a base from which to start. For acute studies, one must rely on past statistics. For example if pneumonia study is being discussed, the CRA will want to know how many patients with pneumonia the Investigator saw over the past year. In either case, the more thorough the records are concerning the patient population, the better the enrollment estimates will be.

There may be several other factors that the CRA will want to discuss regarding enrollment during an evaluation visit. One is whether or not the site is doing, or is planning to do any competing studies within the same time period. A competing study is usually the one in which 
similar subjects are to be enrolled. In order to meet the enrollment targets, it's important that a study does not have to compete with another Sponsor's study.

Another factor is the timing for the study. If the site has too many active studies at the same time, a study may not get the attention it needs to be done well.

CRA may assess the patient flow at site from hospital registry and estimate the number of potential study participants, discuss the proposed recruitment strategies i.e. discussing target dates for starting and completing the study, requirement plan and deliverables from the study, including advertisement, primary and referral sources.

\subsection{Source documentation}

A source document is a document in which data collected for a clinical trial is first recorded. These data are later entered in the case report form. According to GCP, 'Source documents are the Original documents.' Examples of source documents include: Medical records, lab reports, subject diaries, X-rays and ECG printouts. During the evaluation visit, the CRA will discuss the site's source documentation and its practices and archival of source documentation and its accessibility.

The CRA also specifies to the Investigators about his or her obligations to obtain informed consent in accordance with applicable regulatory guidelines and informs that consent documents, signed by the subject will be reviewed to assure that reasonably foreseeable risks are adequately explained.

\subsection{Subject confidentiality}

Subject confidentiality will be discussed by the CRA in detail with the Site staff, as it involves sensitive health information of subject, which after disclosure may create havocs. The CRA will inform the site personnel about their obligations to maintain the subject's confidentiality.

\subsection{Safety}

During the trial, there may be AE/SAEs which may pose risks to the subject's life. During evaluation visit, the CRA will discuss about the Investigator responsibilities and procedures for identifying, collecting, documenting, reporting and following up to the sponsor and to the IEC and any applicable regulatory authorities, adverse events (AEs), serious adverse events (SAEs), pregnancies, medical device incidents or near incidents and the requirements of the protocol

\subsection{Investigational product}

Investigational product (IP) refers to a pharmaceutical form of an active ingredient or placebo being tested as a reference in a clinical trial, including a product with marketing authorization when used in a way different from the approved form, or when used for an unapproved indication, or when used to gain further information about an approved use.

The CRA will discuss the following -

- Brief information and benefits of the IP for the site's patient population

- The investigational status of the test article

- Pl's responsibility regarding IP handling, dispensing, accountability, documentation, and storage conditions; arrangements for collection/ disposal of any used and unused IP

- Security and accessibility to the IP storage area.

CRA will tour the facility for the storage of Investigational product in order to confirm that the facility is well equipped and meet the requirements of the storage conditions prescribed in the protocol.

\subsection{Institutional ethics committee}

All clinical trials carried out at the site must be approved and monitored by the Institutional Ethics Committee, to make sure; the risks are as low as possible and are worth any potential benefits. 
An Institutional Ethics Committee is a committee composed of physicians, pharmacists, nurses, and experts in bioethics, legal affairs, and other experts that guarantees the ethic content of the trial and safeguard the rights of all participants. By law, all institutions performing or supporting biomedical research on humans must have an Institutional Ethics Committee giving its initial approval to a study.

The CRA should be certain that the Investigator is familiar with the Institutional Ethics Committee process and should emphasizes to Investigator his or her obligation to obtain Institutional Ethics Committee review and approval of a clinical investigation before the investigation may be initiated and also to ensure continuing review of the study by an Institutional Ethics Committee and to keep the Sponsor informed of such Institutional Ethics Committee approval and subsequent Institutional Ethics Committee actions concerning the study.

CRA mainly verify with the Investigator on the following:

- Institutional Ethics Committee compliance including composition and working procedure

- Scheduled dates \& frequency of Institutional Ethics Committee meeting and timelines

- Foreseen difficulties in obtaining approval and time for approval

- Anything specifically required by the Institutional Ethics Committee

\subsection{Study management \& administration}

CRA will discuss about study management and administration with Investigator, highlighting the following:

- Expected frequency and duration of monitoring visits

- Site personnel's responsibility

- Adequate facilities provided during monitoring visits

- Responsibility of PI to ensure electronic data system accessed by the Sponsor and its representatives.

- Potential audits and/or regulatory inspections as well as the role of PI and Sponsor during audits

- Management of financial agreements

- Information on key dates such as Investigators' meeting and/or Study initiation meeting, Study drug availability, etc.

- Collection of essential documents

\subsection{Indemnification}

The CRA should discuss about indemnification clause that indemnifies the Investigator and the institution against claims arising from the trial (not including claims that may arise from malpractice and/or negligence by the Investigator or institution).

\subsection{Publication policy}

The CRA should discuss about the publication policy with the Investigator. Generally upon receipt of the written instruction from the Sponsor, Investigator shall have the right to publish the results of his own site patients' data generated during the Study.

\subsection{Finance}

Some preliminary budget discussions can take place at an evaluation visit. The CRA can discuss with the Investigator how the Sponsor prefers to work with respect to the budget and payments for trials. The CRA may also want to find out how the Investigator normally puts together a budget. If the site looks promising, it might be appropriate to talk about the grant range that the company is willing to pay to ensure that both parties are at least in same ballpark. CRA should also discuss the reimbursement plan and costs of tests and procedures for incorporating in the site contract. 
South American Journal of Clinical Research

Volume 3, Issue 1, 2016

\subsection{Essential documents to be collected:}

When the Investigator has been selected they will be required to submit the following documents to the Sponsor:

- Signed Protocol page - it must be signed and dated by the principal Investigator to document their agreement to conduct the trial as per the protocol.

- Signed and completed FDA Form 1572, Statement of Investigator (if the trial is to be filed in support of a US IND).

- Curriculum vitae or CVs of the principal Investigator and related site staff.

- List of Institutional Ethics Committee members and their qualifications to ensure that the Institutional Ethics Committee was properly constituted as per the requirements.

- Laboratory license or certification, laboratory normal values, and a CV for the laboratory director for each laboratory to be used in the trial. If a central laboratory is used, these documents will be obtained directly from the central laboratory by the Sponsor, and copies will be sent to the Investigator site for their files.

\subsection{Site tour}

During evaluation visit, site- facility should he toured to ensure that all of the necessary equipment is available to fulfill the required study procedures. The equipment being used for the trial should be state of the art. The facilities should be clean and orderly, and storage for investigational product must be established. The storage area should be in a secure and locked area or cabinet with access granted only to those personnel assigned by the Investigator. Overthe-counter medications that may also be required for use in a clinical trial should also be stored under the same conditions.

After the site tour; CRA should have the answer to following questions

- Is there secure area and space to store the study drug and other supplies?

- Is the necessary equipment required for the study is present?

- Is there room for a CRA to work during monitoring visits?

- Is the facility clean and well maintained?

- Are there any clinic or hospital policies that would limit the CRA's ability to review source documents, such as patient charts?

\section{Capability of site/ review of the infrastructure requirements}

Conduct of clinical trial requires the presence of certain basic infrastructure at a trial site. CRA reviews the site infrastructure at the time of site evaluation visit and it plays an important role in getting a site selected for a clinical trial.

The basic infrastructure that is required at a trial site includes:

- Space for Storing Study Documents, Study drug and Materials

- Communication facility (phone with STD/ISD facility, fax, internet etc.)

- Local laboratory facility/ Radiological facility (if required)

- Wards/ICUs/ Operation Theatres

- Archival facility

- Standard Operating Procedures

\subsection{Space for storing study documents, study drug and materials}

A potential Investigator/ site is required to have some space dedicated for conducting clinical trial. Each trial will require study specific documentation, which includes, source notes, study records, all the documents filed in the regulatory binder, as well as any study tracking logs, equipment logs, etc. All study specific documents are required to be kept in secure areas, available only to authorized personnel.

This space is required for:

- $\quad$ Storing trial documents; (CRFs, site files, IB, subject files etc.) 
- Storing trial material (Investigational product/comparator, lab kits, biological specimen, packaging material etc.)

- Administration of informed consent and subsequent visits of the subjects (Clinical treatment and examination rooms)

- Management and execution of clinical trial activities by the study team member at the site

- Conduct of clinical trial monitoring by the CRA and Sponsor/CRO representatives

This space should be equipped with:

- Lockable storage cabinets/cub-boards

- Appropriate storage facilities for the archival of study specific documents

- Appropriate storage facilities for storing investigational product/ comparator

- Deep refrigerator for storing biological specimens (if required by the trial)

- Access and environmental control (protection from fire, flood, termite etc.)

- Phone, fax, internet and computer access

- Power back-up

It is not necessary to have all the facilities beforehand as most of the items mentioned above are generally procured from the trial grants. However, there should be a provision to have each one of them as and when required.

\subsection{Communication facility}

A clinical trial site is required to have very good communication facilities. It is essential to have a direct phone line with STD/ ISD facility, fax, Internet etc. in place.

Communication facilities are required for:

- Randomization of patients (either phone or web randomization)

- Transmission of trial data (e, g. ECG tracings, data clarification form, serious adverse event reports etc.)

- Routine communication with CRA as well as study subjects etc.

- Electronic data management (eDM) capabilities; Assess experience, equipment and connectivity and the availability of (internet) access to monitor eCRFs.

\subsection{Local laboratory/ radiological facility}

Safety and efficacy assessment are the primary objective of any clinical trial and for this, a potential Investigator site should be equipped with a good laboratory as well as radiological facility. However, if a site does not have these facilities, it can tie-up with a service provider who is located near to the site. The laboratories should be either accredited by a National/ International accreditation agency or follow the quality control standards laid down for them. Also, there should be a proper access control and a backup mechanism to retrieve data of trial subjects, whenever it is required.

In majority of clinical trials, a central laboratory is used for the purpose of achieving consistency across sites, but the local laboratories are still required for managing routine medical care of the trial subjects.

CRA will have a tour of the laboratory facilities, if either, will be used for the study and assess the availability of:

- Adequately trained personnel: Curriculum vitae(s) of the respective laboratory head

- Equipment and facilities are adequate to perform the necessary protocol specific procedures (e.g. electrocardiogram, spirometer, centrifuge, refrigerator, freezer, )

- Current Accreditation certificate(s) of the laboratories and certification for study period

- Maintenance certificates of the equipment's used in the imaging facility

- Current laboratory normal reference ranges and values for protocol required tests 
South American Journal of Clinical Research

Volume 3, Issue 1, 2016

\subsection{Wards/ ICUs/ Operation theatres}

These are the standard requirements for providing the medical management as well as handling the medical emergencies; however, the requirements may vary across trials.

\subsection{Archival facility}

Since clinical trial documents are required to be retained for a period of 15-20 years, a potential site should be equipped with good archival facility which includes lockable cabinets/ cub-boards and environmental control (protection from fire, flood, termite etc.).

Archival facility is required for:

- Storing the trial documents for a specified duration after the completion of a trial

- Preserving the trial documents for regulatory inspection/ audits

- Ensuring compliance with applicable regulatory requirements

\subsection{Standard operating procedures (SOPs)}

Schedule-Y of Drugs and Cosmetics Act, requires every Investigator site to have documented Standard Operating Procedures for the task performed by them. SOPs are detailed, written instructions to achieve uniformity in the performance of a specific function.

Typical SOPs for an Investigator site should include following topics:

- Preparation, review and approval of SOPs

- Constitution of study team

- Feasibility assessment

- Institutional Ethics Committee submission

- Study initiation, execution, and completion

- Delegation of duties

- Administration of Informed Consent Form

- Reporting of Serious Adverse Event(s)

- Management of investigational product

- Record retention, update and retrieval

- Communication flow between team members

- Archival etc.

\section{Documentation and reporting}

The CRA should be sure that a thorough evaluation of the site has been made before leaving the site and notes have been made for future reference.

At the completion of the evaluation visit, the CRA will complete a written evaluation visit report and document the findings in the comment section and submits the same to the Manager for his review and comments. This report will document any observations, discussions or findings from this visit in a standard format as well as any agreements reached by the Investigator and/or staff. All information gathered from this visit will be reviewed by the Sponsor and based on the evaluation visit report, a decision is made to include or exclude this Investigator/ site.

At the end of the all the evaluation visits to all the potential sites identified for a particular study, the Sponsor finalized the sites, which qualifies to the requirement of the study Sponsor.

Some of the major factors that are considered while selecting among the sites is as follows:

- Experience in clinical research and human clinical trials of the disease under study.

- Location of the site: is it in an area that is easily accessible to subjects? Is it in a center of excellence for the therapeutic indication under study?

- Does this Investigator have the appropriate subject population available to them to be able to recruit study subjects for the trial in a timely fashion?

- Is the budget proposal from this Investigator appropriate or is it cost-Prohibitive? Does the institution require an overhead cost that is beyond budgetary restrictions?

- Can they complete the trial in the given time frame? 
- What methods will they have to employ to recruit subjects? Will they require extra assistance to recruit subjects?

- Does the Investigator have the appropriate staff available including a clinical research coordinator to assist with the conduct of the trial? Does the staff have the appropriate education and experience to conduct the trial?

- Have the Investigators ever worked with the Sponsor in the past and if so, how was their conduct during the course of the trial?

- Do the Investigators have any other current research commitments that would compete with the trial you are trying to place at their site?

- Has the Investigator been previously inspected by the US FDA or another regulatory authority and if so, what was the outcome of that inspection?

The sites, which do not qualify to the requirement of the study due to inadequate / improper facilities and infrastructure, these are upgraded if Sponsors budget has the provision for the same. If the sites do not qualify with regards to capability of the Investigators or any ethical considerations, these sites are not selected for the study. However, in either case, the Sponsor should take up the moral responsibility to inform the site appropriately. Hence it is appropriate to send a thank you letter to the site within a few days of the visit. If the Investigator is selected to do the study, a telephone followed by a confirmation follow-up letter will be sent to the Investigators informing them of this decision.

A copy of site evaluation visit report is archived in the master files

\section{The process flow during the site evaluation activity is as follows}

Whenever a new investigator site is being approached for participation in a clinical trial, the first step is an execution of a Confidentiality agreement (CDA)/ Non-Disclosure Agreement (NDA) between the Sponsor/CRO and the Investigator. This agreement establishes the obligation on the part of investigator for maintaining the confidentiality of information being provided to him/discussed with him by the Sponsor/ CRO designee. Once CDA/ NDA is executed, Sponsor/CRO designee forwards a study feasibility questionnaire/ protocol synopsis/ brief study outline to the Investigator or discusses it over phone. If the response of the investigator on the study feasibility questionnaire meets the expectations of the Sponsor/ CRO, a site evaluation visit is organized at the investigator site. Based on the outcome of the visit, a new investigator site is either selected or rejected for participation in a clinical trial. However, if the site meets all the pre-requisite requirements it is highly unlikely that it gets rejected.

\section{Conclusion}

In conclusion, both sites and Sponsors benefit from intentional relationship building, especially during the study startup phase. Building good relationships can lead to positive and productive interactions and successful trials with a minimum of stress. The process should include key strategies, such as clear and meaningful communication, transparency in the data being exchanged, and flexibility to adapt to the ever-changing flow of the study during all of its phases. Being mindful of these strategies and accountable for their implementation can contribute to the success of a trial that goes far beyond a metrics spreadsheet.

\section{References}

[1.] Andrea G. Procaccino. Managing Clinical Trials. New Drug Approval Process

[2.] Carol Opalek \& Joy Frestedt. 2009. Study Startup 101. The Monitor

[3.] Fundamentals of Clinical Research. 2007 edition.

[4.] Dr. Julka P. K. Becoming a Successful Clinical Trial Investigator.

[5.] Karen E. Woodin. Coordinating Clinical Research

[6.] Karen E. Woodin \& John C. Schneider. Monitoring Clinical Research; Second Edition.

[7.] Dr. Umakant Sahoo. Monitoring Visits. Monitoring and Source Data Verification in Clinical Trial Management 
South American Journal of Clinical Research

Volume 3, Issue 1, 2016

\section{Important websites}

[1.] http://www.ich.org. ICH Guideline for Good Clinical Practice on Good Clinical Practice [2.] http://www.cdsco.nic.in/html/GCP.html. Good Clinical Practices for Clinical Research in India

[3.] http://www.cdsco/nic/in/html/D \& C_Rules_Schedule-Y (Amended version 2005)

[4.] www.fda.gov

\section{Authors profile}

\section{Vinod Gyanchandani, MBA}

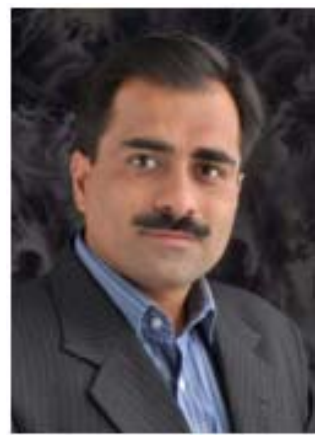

Vinod serves Global Drug Development Experts as Country Head- Clinical Operations. Vinod holds a Master's in Business Administration and a Bachelor in Pharmacy and pursuing Ph. D in Clinical Research from Texila American University.

During a career in pharmaceutical industry spanning 18 years, Vinod started his professional career with GlaxoSmithKline in Sales \& Marketing and finally decided to enjoy the exciting world of clinical development and made clinical research as a core area of interest. Vinod has been in the field of clinical research for over 10 years now and has expertise in almost all major therapeutic areas in different capacities viz. Asst. CRM, Sr. CRA, Clinical Research Manager at GlaxoSmithKline.

In current profile, Vinod heads finance, business development, human resource and clinical operations in India. His team is involved in phase I to IV clinical trials. He is passionate about clinical project management, and likes to develop, refine and implement the classic project management principles for successful conduct of clinical trials.

Vinod has been a DIA member for about 6 years and lifetime member of Indian Society for Clinical Research.

Vinod resides in Nagpur with his wife Priya, sons Yash \& Harsh. Vinod enjoys swimming, reading and playing chess and regularly contributes his time and resources to provide medicines and educational material to needy people via various NGOs. 